\title{
PLANTAR NUEVAS IDEAS EN EL MUNDO EDITORIAL ACADÉMICO. EL REDISEÑO DE LA REVISTA SAPIENTÍA DE LA UNIAJC
}

\section{PLANT NEW IDEAS IN THE ACADEMIC PUBLISHING WORLD. THE REDESIGN OF THE UNIAJC MAGAZINE SAPIENTÍA}

\author{
Mónica Peláez Montoya, Keyla Tatiana Tejada Pineda, Angie Melissa Tobar Bolaños y Lina Marcela Tejada \\ Semillero Alografía \\ Institución Universitaria Antonio José Camacho \\ Cómo citar este artículo: \\ Peláez Montoya, M., Tejada Pineda, K.T., Tobar Bolaños, A.M. y Tejada Pineda, L.M. (2021). Plantar nuevas ideas en el mundo editorial \\ académico. El rediseño de la revista Sapientía de la UNIAJC. Revista Sapientía, 13(26), 32-42.
}

\section{EL TERRENO Y LA SIEMBRA}

Esta revista que estás leyendo, es también llamada en el ámbito editorial, publicación o revista de divulgación académica $^{1}$. Pertenece a la categoría de revistas de investigación ${ }^{2}$ o revistas científicas ${ }^{3}$. Es una pieza de comunicación que ha llevado, de parte nuestra en el semillero, un proceso de diseño editorial iterativo ${ }^{4}$ (Figura 1), que sintetizamos de la siguiente manera: en primer lugar, realizamos una caracterización de la categoría de revistas y un diagnóstico de las publicaciones de la universidad. Seguido identificamos los elementos fundamentales del diseño editorial que necesitábamos en este tipo de publicaciones para iniciar a diseñar, o mejor, a rediseñar, pues ya existía la revista "Sapientía" en la universidad desde hace algunos años. Cuando supimos lo esencial para tener en cuenta, como el posicionamiento de marca, la anatomía y la percepción de los usuarios (estudiantes y profesores); empezamos a profundizar en la búsqueda de un nueva personalidad para la marca y una paleta de estilo que pudiera identificarla, guardando un equilibrio con los lineamientos gráficos institucionales. Una vez tenidos en cuenta estos detalles, iniciamos a crear un Storyboard para encontrarle un ritmo y una composición a las páginas. Maquetación que pudiera mediar la función de comunicación de la revista y los intereses de los lectores potenciales. De esta forma iniciamos la concreción del prototipo.

\footnotetext{
${ }^{1}$ La revista de divulgación de carácter académico es una publicación periódica que tiene como componente principal el artículo científico. Tiene un nombre distintivo, se publica a intervalos regulares y cada entrega está numerada o fechada consecutivamente (Hernández, 2010). Es importante agregar que las revistas de divulgación académica que buscan ser catalogadas en los índices de clasificación deben reunir algunas condiciones formales adicionales que no están contempladas en los libros tradicionales sobre publicaciones impresas (Benavides, 2013).

${ }^{2}$ Las revistas de investigación son publicaciones seriadas que cuentan con ISSN, pueden ser físicas o virtuales, deben cumplir con las exigencias mínimas del escalafón C del sistema nacional de indexación y homologación de revistas especializadas de CT+I Publindex de COLCIENCIAS (Benavides, 2013).

${ }^{3}$ Una revista científica puede definirse como una publicación seriada y científica que incorpora resultados de procesos de investigación (Herrera, Paz Enrique \& Hernández Alfonso, 2018). Las revistas científicas son el medio más rápido para conocer los últimos avances sobre un campo específico del conocimiento. Son consideradas como los eslabones básicos en el proceso de transferencia y difusión de la ciencia y un instrumento privilegiado para la comunicación entre los investigadores (López y Cordero, 2005). Guillamón (2006) afirma que el objeto de las revistas científicas es la discusión abierta y libre de hallazgos experimentales, hipótesis y teorías. Esto implica que la revista no es simplemente un repositorio de información, sino que es el medio de comunicación que permite a los investigadores "influir con el propio conocimiento en el núcleo de investigadores internacionales de su especialidad". Las publicaciones son un indicador de la competencia entre académicos e investigadores (Alfonso, 2010).

${ }^{4}$ Diseño iterativo: es el proceso vinculado a los métodos de investigación en diseño y se define como un ciclo de creación de prototipos, pruebas, análisis y depuración. En este tipo de diseño, el proyecto creativo se evalúa con métodos de investigación basados en el usuario y se realiza comparando las versiones sucesivas o iteraciones con las metas del proyecto. La complejidad de las iteraciones dependerá de qué tácticas de investigación se utilicen y de cuántas iteraciones se pretenden realizar. Si se trabaja en un rediseño se debe dar tiempo para realizar pruebas con los elementos originales antes de iniciar los ciclos de iteración nuevos. Dado que la investigación orientada al diseño es de naturaleza cualitativa, es raro que los prototipos satisfagan a todo el mundo y es por esto por lo que los resultados de las pruebas deben ser coherentes con los objetivos del proyecto. Las pruebas son para obtener información no para paralizar el proceso o predecir resultados (Visocky, 2018)
} 


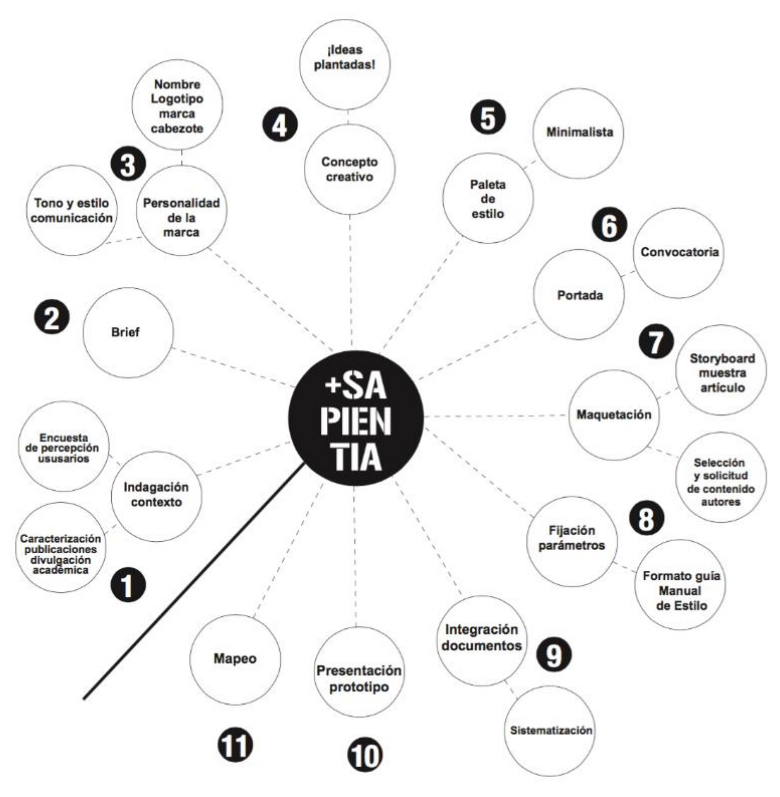

Fig. 1. Mapa del proceso de diseño. Rediseño de la Revista Sapientía. Fuente: (Semillero Alografía, 2021)

Claro está que, para gestionar estos procesos, nos debimos acercar a la indagación exhaustiva de documentos referentes de proyectos, categoría de revistas, tipos de marca, composición de revista y estilística, etc. También nos apoyamos con la realización de una encuesta de percepción de marca y de categoría. Como con la ideación o lluvia de ideas, a través del listado de palabras y la cartografía mental (Buzan, 1994) o Mind Maps y el estabecimiento de un Brief. Tomando decisiones a través de los insights ${ }^{5}$ hallados y prototipos ${ }^{6}$ creados para tales fines.

Así, iniciamos a investigar integrando en el proceso, principios y herramientas del Pensamiento de diseño o Design thinking: empatizar, definir, idear, prototipar $y$ testear (Brown, 2008). Para enmarcarnos proyectivamente en las etapas generalizadas de la Investigación Basada en Diseño (IBD): Investigación preliminar, desarrollo y pilotaje, evaluación final (Romero-Ariza, 2014). Adoptando a su vez metodologías activas-colaborativas para facilitar las dinámicas del trabajo y los procesos de aprendizaje en el semillero.
Habiendo dado paso a la anterior descripción, a continuación, queremos profundizar en forma reflexiva, un poco más sobre el proceso, ampliando el propósito de este artículo que incluye tener en cuenta las experiencias, motivaciones y aprendizajes de las integrantes del equipo de investigación. También las implicaciones del Diseño Visual y los elementos metodológicos que hacen parte. Para dar cuenta, finalmente, de algunos resultados parciales de esta primer etapa de la investigación en la que estamos.

\section{LAS CUESTIONES DE SER DISEÑADORAS VISUALES SEMILLERISTAS}

El poder realizar estudios en Diseño Visual ha creado en nosotras la necesidad de querer transformar el mundo que está a nuestro alrededor. Pensar en cada circunstancia nos ha motivado a poder visualizar y establecer soluciones para mejorar la calidad de vida de las personas.

Siempre hay una generosidad implícita al pensar como diseñadoras. Es el querer estar en el lugar del otro y procurar crear las circunstancias para construir un mundo mejor. Estamos de acuerdo con el pensamiento de Frascara (2005): [...]"se reconoce a la función del diseño en lo que respecta a posibilitar la vida y también para mejorarla. Para ayudar a la gente a descubrir diferentes dimensiones de la cultura y el placer". Agrega que hoy día, socialmente, el acceso a la información debería verse como un derecho y en esto los diseñadores visuales tenemos una gran responsabilidad.

\footnotetext{
${ }^{5}$ Insights: uno de los elementos esenciales del Pensamiento de Diseño o Design Thinking. La búsqueda de insights se define como la exploración de nuevas oportunidades a partir de indagar con profundidad en nuestros (futuros) clientes, usuarios, consumidores o públicos. Es, en pocas palabras, descubrir el por qué la gente elige lo que elige. Por qué la gente elige ese tipo de solución y no otra. Ese por qué debe ser distinto, interesante, novedoso. Un insight es un insight cuando se descubre algo que hasta el momento no había sido descubierto (Kastika, 2021)

${ }^{6}$ Prototipos: uno de los elementos esenciales del Pensamiento de Diseño o Design Thinking. Los prototipos se usan para el testeo rápido de ideas, que nos permiten experimentar, aprender, generar nuevos insights y seguir profundizando en la innovación. Así, lo más importante de las ideas es probarlas lo más pronto posible y llevarlas a una buena escala. Es decir, no se espera a que la idea esté totalmente desarrollada para realizarlo, sino que se hacen pausas para probar y testear. Se aprende de lo que se dice de ello y se vuelve a mejorar la idea. Hasta que se llega a una especie de prototipo óptimo esperado (Kastika, 2021).
} 


\section{4 | Sapıentia Vol. 13, №. 26, noviembre 202I. pág. 32-42 ISSN I909-08II}

El pertenecer a un semillero de investigación ha permitido que, desde el papel de estudiantes, contemos con la posibilidad de empezar a poner un granito de arena para realizar procesos de diseño que ayuden a entender o transmitir conocimientos útiles, aportando valor a la comunidad educativa. Nos ayuda a visualizar un panorama más amplio de nuestra profesión, nos brinda la seguridad para crear nuevos escenarios, generar nuevas ideas y un mayor aprendizaje interdisciplinario y colaborativo con toda la comunidad.

La generación de nuevas ideas trae consigo entender estos diferentes procesos, como la metáfora de una siembra de la cual somos semillas. No por nada somos llamadas "semilleristas". Estas ideas y conocimientos, que son como pequeñas semillas, deben encontrarse con un buen terreno, una tierra abonada por la educación, la investigación y la curiosidad. A este hermoso tipo de suelo, lo podemos llamar universidad, para posteriormente ir regando, nuestra semilla, con gotas de consistencia, trabajo metodológico y el acompañamiento docente. Pero también tenemos elementos externos que no vienen de nosotros, como el sol o la lluvia. Estos son elementos externos que pueden ayudarnos a crecer o no, si no tenemos cuidado. A veces pueden ser la distancia, prevención, poco conocimiento o la percepción inadecuada del tema, lo que puede afectar su crecimiento. Por último, tenemos el elemento del tiempo. Al inicio cada tema de investigación no es más que una pequeña semillita, pero con el tiempo puede evolucionar en una hermosa planta que da sus propios frutos. Ver la evolución de una pregunta en una tesis, en el trabajo de campo con un grupo focal o en la creación de un producto de investigación, es un acto mágico que sólo el tiempo y la constancia pueden hacer realidad.

Así mismo funcionan los procesos de investigación, como establece Sabino (1992) existen cuatro momentos o fases en la investigación que, desde un punto de vista abstracto, muestran las sucesivas acciones que va desarrollando el investigador mientras trabaja, estas son: momento proyectivo, momento metodológico, momento técnico y momento de síntesis. En otras palabras, es necesario desarrollar un sistema que permita cumplir, al ser conscientes de cada uno de estos pasos, para poder desarrollar un buen proyecto.
Todo esto nos conduce a un proceso de auto-cuestionamiento, donde comenzamos a indagar la relación del objeto de estudio, el público objetivo y los procesos caracterizados según el contexto, en este caso el universitario.

En nuestra experiencia actual, en el semillero Alografía, trabajamos en el rediseño de la revista Sapientía perteneciente a la Facultad de Ciencias Sociales y humanas, cuya función comunicativa es dar a conocer artículos de investigación derivados de los proyectos de investigación propuestos por los semilleros de investigación de la UNIAJC. Y que, además, tiene como objetivo el fomentar el amor por la investigación en los estudiantes universitarios, generándoles oportunidades de vinculación a través de sus propias prácticas.

Debido a esto y en medio del camino, nos han surgido varios cuestionamientos que esperamos ir resolviendo. Algunos de ellos son: ¿Cómo hacer más digerible para los estudiantes universitarios los artículos científicos? ¿Cómo acercar más a los estudiantes universitarios al gusto por la investigación? ¿Cómo acercar más a los estudiantes universitarios al gusto por las revistas de divulgación? ¿Cómo humanizar este tipo de contenidos?¿Cómo se educa a los estudiantes para la lectura del material académico?

El proceso para responder estos cuestionamientos nos ha hecho comprender la importancia del saber encontrar referentes de estudio como: artículos, investigaciones, libros, autores, proyectos de tesis, métodos, productos, entre otros. Pero no solamente es el hecho de encontrar esta información que nos será útil, sino también, la forma correcta de entenderla y tener acceso a los lugares más apropiados para conseguirla. Pues, en nuestra experiencia como estudiantes, hemos evidenciado la dificultad por parte de algunos compañeros para encontrar, comprender o poder analizar correctamente estos documentos. La falta de entendimiento o enfoque del hallazgo y la comprensión lectora de los temas indagados, en un principio, puede alejarlos del gusto por la investigación. Otro factor que hemos detectado es la percepción lejana de lo que es la dinámica de la investigación en la universidad, por la poca información que llega a los oídos de los estudiantes o que se comparte al respecto. Es por ello que la posibilidad de pertenecer a un semillero o a un proyecto de investigación como estudiante puede ser muy baja. 
De acuerdo a lo anterior, al detectarse una carencia en la dinámica académica y de producción de conocimiento generalizada de parte de los estudiantes, el Decanato Asociado de Investigaciones de la UNIAJC ha querido promover en la comunidad educativa el fortalecimiento de esta publicación para la difusión y formación de nuevos semilleros de investigación. Así, desde el programa de Diseño Visual, en conjunto con el Comité Editorial de la Facultad de Ciencias Sociales y Humanas y el Semillero Alografía, nace la idea de rediseñar la revista Sapientía, partiendo de la necesidad de acercar la información en una forma más directa, efectiva e interesante para los estudiantes y, de esta forma, se puedan relacionar directamente con los procesos de investigación que se realizan dentro de la universidad.

\section{LOS TIPOS DE ABONO}

\section{El diseño visual en el campo investigativo}

Jorge Frascara (2005) propone al diseño como una disciplina dedicada a la producción de comunicaciones visuales dirigidas a afectar el conocimiento, las actitudes y el comportamiento de la gente. En esta definición la gente asume un rol central, y las decisiones visuales involucradas en la construcción de mensajes no provienen ya de supuestos principios estéticos universales o de caprichos personales del diseñador, sino que se localizan en un campo creado entre la realidad actual de la gente y la realidad a la cual se desea arribar después de que la gente se encare con los mensajes. Por eso en nuestra propuesta de diseño editorial tenemos en cuenta tanto las necesidades, percepciones y deseos reales de los usuarios acerca de la revista y su visualización.

Procuramos que la información contenida en la revista pueda ser asimilada y comprendida por los lectores. Respecto a esto, el autor agrega que, para que las comunicaciones puedan afectar el conocimiento deben ser construidas sobre la base de un buen conocimiento de la percepción visual y el sistema de valores del público al que se dirigen, entre otras. Insiste en considerar siempre la percepción y los códigos visuales que los usuarios tienen y que pueda relacionarse con el producto y ser asumida por un experto o profesional. Esto nos recuerda, además, la necesidad de conocer profundamente a los usuarios que propone el Pensamiento de Diseño para llevar a cabo propuestas innovadoras.

Generalmente en los procesos de investigación emergen tensiones en variados aspectos $\mathrm{y}$, esta dinámica, pone en consideración desde las relaciones personales hasta las relaciones del diseño mismo con quienes lo piensan y lo practican. Además de la relación para quienes se trabaja o quienes van a ser impactados o recibirán algún beneficio con los resultados. Debido a esto abordamos el tema de la ética desde el diseño como comunicación visual.

Dentro del campo investigativo, los diseñadores visuales entran a considerar responsabilidades éticas. Al ser parte de un sistema de comunicación humana, en el reconocimiento del Otro -el receptor de la comunicación- como sujeto (una persona) y no como objeto (Frascara, 2005, p.48). Es decir, se vuelve necesario establecer mecanismos de comunicaciones más humanas en donde el productor de la comunicación debe tratar de hablar el lenguaje de la audiencia.

Las comunicaciones que no ofrecen espacio para la interpretación o para la construcción de posible respuestas individuales promueven reacciones extremas. De aquí que ciertas categorías de piezas de comunicación visual, como las editoriales, puedan estar desconectadas del interés de los lectores.

En este sentido, Frascara propone una noción de co-autoría desde el punto de vista ético. Lo que nos recuerda la noción de Diseño Colaborativo o Co-Design (Sanders, 2008). Explica además que es en las situaciones de asociación en donde las relaciones se hacen éticas, donde los talentos rinden frutos, donde se pueden realizar proyectos complejos y ambiciosos y donde los diseñadores pueden desempeñar el papel de catalizadores y colaboradores en la creación de un ambiente cultural y conceptual en constante desarrollo (Frascara, 2005, p.51).

Vemos cómo los diseñadores visuales como investigadores, en el desarrollo de productos con énfasis en la responsabilidad social, empiezan a concebir su trabajo 
más como estrategas de la comunicación que como simples dibujantes (Frascara, 2005;p.58).

\section{EL REDISEÑO DE LA REVISTA SAPIENTÍA}

Caldwell y Zapaterra (2014) señalan que "La mejor razón para acometer un rediseño es la de mantenerse en sintonía con los lectores y reflejar sus necesidades" (p.198). Asimismo, hacen énfasis en que, para asegurar un sostenimiento de una publicación que esté dirigida a jóvenes o adolescentes, deberá estar más atenta a las tendencias y transformaciones culturales en las que se ven insertos, para poder reflejar su realidad. En nuestro caso, esto debe tenerse muy en cuenta, pues la revista actual busca dirigirse directamente a los estudiantes universitarios. Encontramos en esto un apoyo para el rediseño de la publicación y la atención al mismo.

Apoya esta noción la propuesta de Benavides (2013), que señala dos aspectos para el mejoramiento de la calidad de una publicación: el contenido y la forma. Es decir, no solamente debe existir preocupación por los requerimientos del contenido de esta categoría de publicaciones, sino también por la coherencia y calidad de su representación gráfica. Sobre el primer aspecto, las editoriales tienen estrategias muy claras para velar por su calidad: el arbitraje por parte de expertos y la revisión ortotipográfica ${ }^{7}$ y de estilo ${ }^{8}$ por parte de un corrector. Hacen lo propio, en este caso, los comités editoriales institucionales. Existe, además, la evaluación de la comunidad académica que, aunque está fuera del dominio de la editorial universitaria, ejerce tal vez el control de calidad más importante en su condición de lector-consumidor (Benavides, 2013). Es por esto que el testeo de los prototipos con los usuarios (comunidad universitaria) sigue cobrando importancia.

\section{DISEÑO EDITORIAL}

El área de Diseño Visual en la que estamos enfocadas es el Diseño Editorial. De esta forma y, antes que nada, debemos acercarnos a la definición del Diseño Editorial que compartimos desde la mirada de Cath Caldwell y Yolanda Zapaterra (2016), quienes lo consideran como una forma de periodismo visual, rasgo diferencial de otras disciplinas del Diseño Visual y de algunos formatos interactivos. Es decir, los diseñado- res visuales entran al campo editorial para formarse dentro del campo de la comunicación con una consciencia más centrada. Las publicaciones editoriales de comunicación como educar, entretener, persuadir, etc.; o bien ser una mezcla de lo anteriormente señalado. Con el rediseño de Sapientía, que es principalmente una revista educativa, se propone abrir su función comunicativa hacia la difusión de información como: oportunidades de investigación que los estudiantes puedan tener en semilleros de otras universidades, información de intercambio, alianzas, beneficios académicos, tips para formarse como investigadores y en general potenciar el movimiento estudiantil interinstitucional.

Diseñar o rediseñar una pieza editorial implica tener en cuenta algunos elementos. En tal sentido, lo primero que se debe establecer en una publicación nueva o que necesite un rediseño es el mensaje de marca, es decir, su identidad (Figura 2), su forma de expresarse y las emociones que desea transmitir (Caldwell y Zapaterra, 2014, p.42).

\footnotetext{
7 Ortotipografía. Conjunto de normas que rigen la correcta composición tipográfica. (Aunque suele definirse como normas de la ortografía aplicadas a la tipografía o a lo impreso, tal definición no sólo resulta inexacta -pues excluye aspectos privativos de la composición tipográfica- sino también inútil, ya que, si bien la palabra ortografía alude a lo manuscrito, en ella se ha incluido -desde hace mucho tiempo- implícitamente lo impreso, tanto en gramáticas como en diversos estudios de lingüística. En la práctica, de hecho, la ortografía rige toda manifestación grafémica de la lengua, cualesquiera que sean los medios utilizados para ello, por lo cual bastaría, en todo caso, redefinir la ortografía como "conjunto de normas que regulan la correcta escritura o realización grafémica de una lengua). (López, 2009)
}

${ }^{8}$ Estilo. En otras palabras, el 'estilo' es la manera en que una idea es expresada y que manifiesta una determinada estructura constante e intencional, que puede coincidir o no con las Formas habituales de la lengua. De hecho, el 'estilo' está indisolublemente ligado al tema y a la idea que se pretende manifestar, de modo que, "si se altera ligeramente la expresión, se altera levemente la idean, pues "cuando un autor corrige y pule su estilo, está corrigiendo la idea también.

El 'estilo' en un texto, pues, es el resultado de una serie de elecciones a partir de las posibilidades que brinda la lengua, y son tres los aspectos esenciales que lo conforman: la sintaxis, el léxico y la puntuación. El orden en que se combinan las palabras para formar frases y oraciones que a su vez constituyen enunciados es, ciertamente, una decisión autoral, pero en ello también interviene la labor del corrector o editor. (López, 2009). A de aclarar que esta definición hace referencia al estilo vinculador a lo lingüístico, pues la para el ámbito gráfico también es una palabra que se adopta para describir el lenguaje visual y sus elementos, en los procesos del Diseño Editorial. 


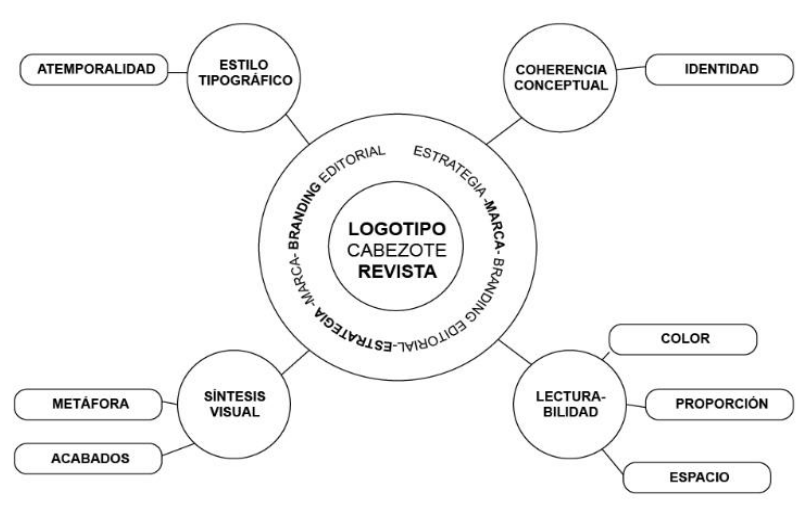

Fig. 2. Elementos del Brandig Editorial Fuente: (Peláez, 2021).

Se entrevé la necesidad entonces de que los elementos gráficos de una publicación (logotipo o cabezote, paleta de color y estilo, anatomía, retículas compositivas, tipografía, fotografía e ilustración) estén sujetos a una regulación a través de una serie normas, generalmente reunidas en un Manual Editorial.

Todos estos elementos constituyen la identidad visual de la publicación. Así cada vez que la publicación emita una nueva edición, los elementos deben ser supervisados para que se le pueda dar continuidad y garantía al dinamismo, al sostenimiento de los valores y la personalidad de marca establecidos, sin poner en riesgo que su contenido, pueda volverse monótono y predecible. Es por esto fundamental lograr que el estilo de la publicación resulte reconocible a los usuarios directos o potenciales, y permanezcan siempre los temas asociados a sus intereses.

En la dinámica de un semillero de investigación basado en el Diseño Editorial, se presume el desarrollo de competencias, habilidades disciplinares y atributos indispensables del diseñador editorial, que entran a desarrollarse en la práctica (Tabla. 1):

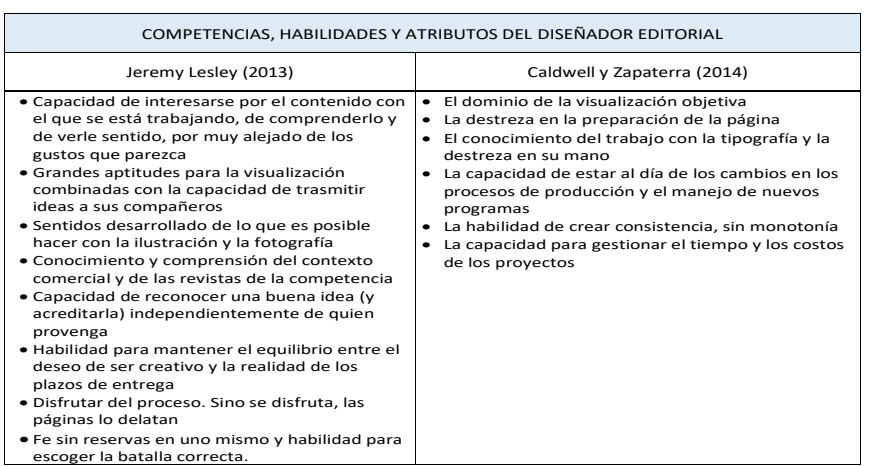

Tabla 1. Competencias, habilidades y atributos del diseñador editorial. Fuente: elaboración propia

\section{HERRAMIENTAS PARA LA SIEMBRA}

\section{Metodologías}

Para poder sembrar todas estas ideas, debemos tener en cuenta varios aspectos del terreno que están implicados y que debemos conocer, observar, facilitar y cuidar en diferentes momentos y procesos, mediados a su vez por métodos de aprendizaje y dinámicas grupales para gestionar la información de los resultados que van surgiendo. En el mapa de las dinámicas metodológicas (Figura 3), que expondremos brevemente a continuación, se muestra la forma en que se interrelacionan.

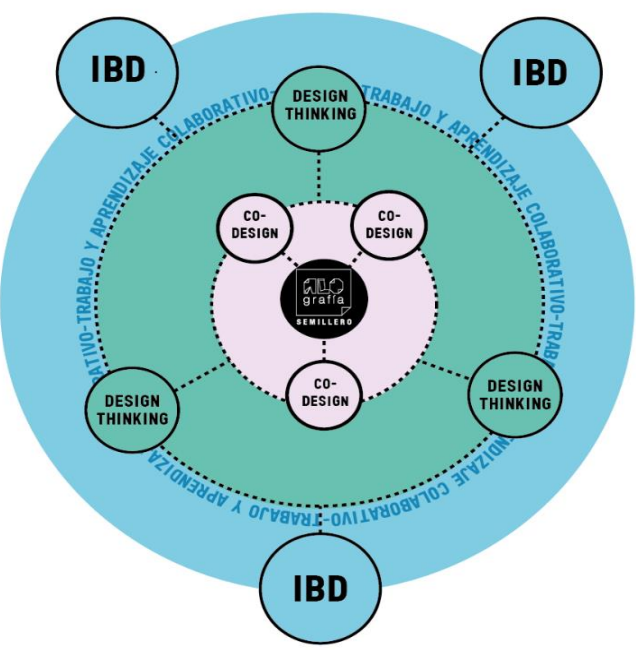

Fig. 3. Mapa de dinámica metodológica Fuente: elaboración propia Semillero Alografía.

\section{INVESTIGACIÓN BASADA EN DISEÑO}

Lleva a cabo experimentos de diseño que buscan desarrollar la ciencia de diseño, que puedan guiar el desarrollo de ambientes de innovación en la práctica. También identifica variables que pueden influenciar su éxito o fracaso. Propone estrategias que integren un enfoque holístico, creación y evaluación iterativa de intervenciones complejas en aulas reales, colaboración entre investigadores y docentes, además, genera procesos constructivos, acumulativos, autorregulados y colaborativos de aprendizaje (Corte, 2009).

Consta de las siguientes características: la decisión de ubicar la investigación en el contexto natural, el propósito de producir cambios específicos en ese contexto; la opción por los enfoques sistémicos y el carácter cíclico e iterativo de los diseños. Es un tema de investigación relativamente reciente, con un abordajecognitivo del aprendizaje, relacionado con formas de aprendizaje académico independientes y efectivas que implican metacognición, motivación 
intrínseca y acción estratégica (Gibelli, 2014).

Según Romero-Ariza (2014) existe un consenso generalizado sobre las etapas que conlleva asumir esta línea investigativa:

\section{Investigación preliminar}

Implica el análisis de necesidades y la descripción del problema, así como la revisión de la literatura especializada, con el objeto de identificar trabajos previos con un propósito o énfasis similar, así como establecer la fundamentación y el marco teórico de la investigación.

\section{Desarrollo y pilotaje}

Supone la elaboración, revisión y mejora progresiva de prototipos con base en estudios sistemáticos tras sucesivos ciclos de investigación. El elemento clave que orienta la mejora es la evaluación formativa resultante de cada iteración.

\section{Evaluación final}

Su principal finalidad es valorar si la intervención o el producto final satisface los objetivos y requerimientos planteados en principio. Puesto que esta etapa suele integrar recomendaciones para la mejora, algunos autores la denominan evaluación semisumativa (Plomp, 2013, citado por Romero-Ariza; 2014, p. 163). Esta fase incluye el análisis y la reflexión sistemática destinada a la obtención de conclusiones que orienten futuros diseños.

En este sentido, la investigación Basada en el Diseño se diferencia de otros paradigmas investigativos en las formas en que se problematiza el contexto y las intervenciones. Aportando validez en los hallazgos encontrados en las asociaciones e iteraciones, que dan como resultado una mayor alineación de la teoría, el diseño, la práctica y la medición a lo largo del tiempo (The Design-Based Research Collective, 2003).

\section{PENSAMIENTO DE DISEÑO}

También conocido como Design Thinking (Brown, 2008), es una disciplina que usa la sensibilidad y los métodos del diseñador para hacer coincidir las necesidades de las personas. Es un proceso para estimular la innovación, basándose en métodos de ingeniería y diseño combinándolos con ideas de las artes, las ciencias sociales y el mercado. El proceso del pensamiento de diseño parte de un contexto y todos los elementos que están inmersos en él, para que, a partir del entendimiento de estos y el surgimiento de insights, se comience a complejizar logrando un alcance máximo de abstracción y análisis, para posteriormente comenzar con la fase de propuesta de estrategias y desarrollo de "prototipos", que ayudan de una manera efectiva en la prueba de las soluciones a implementar. Esta metodología nos aporta herramientas útiles para la generación de ideas y para la gestión de los procesos de diseño.

\section{DISEÑO COLABORATIVO}

Cuando se participa en el Diseño Colaborativo o Co-design (Sanders, 2014) se lleva la creación conjunta a la práctica, para cambiar el cómo diseñamos, qué diseñamos y quién diseña.

Los campos del diseño y la investigación del diseño continúan cambiando a medida que el diseño y la investigación se difuminan. Prácticamente, el diseño puede convertirse en sinónimo de investigación, creando nuevas oportunidades para diseñadores $\mathrm{e}$ investigadores. Es por esto que la investigación se está volviendo más prominente en los planes de estudio de los programas de diseño, así como en el fortalecimiento de los vínculos entre las ciencias sociales.

Los equipos de co-diseño son mucho más diversos hoy en día. A futuro el co-diseño estará en estrecha colaboración con todas las partes interesadas en el proceso de desarrollo del diseño, junto con una gran variedad de profesionales con habilidades híbridas de 
diseño-investigación. Estos “jugadores” conformarán simultáneamente un equipo con muchos tipos de cultura: cultura disciplinaria, cultura de la empresa, cultura étnica, visión del mundo, mentalidad, etc. Razón por la cual, a futuro, serán altamente valorados los nuevos lenguajes, que el co-diseño apoya y facilita, dentro de las variadas formas de comunicación intercultural (Sanders, 2014).

Se co-diseña identificando a los co-diseñadores con quién colaborar, según el tipo de proyecto y las disciplinas que puedan estar implicadas; se define la cuestión a través de una pregunta de investigación concreta, pero suficientemente abierta como para no anticipar la solución; se crea un kit de co-diseño con herramientas que inviten a los participantes a vincularse activa y creativamente en el proceso; se escucha y se interpreta, se reflexiona y se aprende de la implicaciones, expectativas y deseos de la gente (Lupton, 2017).

\section{APRENDIZAJE Y TRABAJO COLABORATIVO}

Derivado de la acción conjunta realizada en el semillero, nos encontramos con un grupo de trabajo colaborativo. Una situación se denomina como colaborativa cuando las personas están en simetría, tienen alguna meta en común y hay bajo nivel de división del trabajo (Delgado, 2015).

En la división del trabajo colaborativo, los cargos o responsabilidades tienen que estar altamente entrelazados: una persona monitorea a otra. En el trabajo cooperativo, la subtareas son independientes. En el aprendizaje colaborativo, la división horizontal de la labor es inestable. Los roles pueden variar cada pocos minutos, transformándose el regulador en el regulado, mientras que la cooperación se refiere a una división más fija del trabajo, usualmente explícita desde el comienzo. (Delgado, 2015, p.27).
Los grupos que trabajan colaborativamente guardan ciertas características y desarrollan ciertas habilidades. Para Rafael Gonzáles (1996) se ha de tener en cuenta que:

- El liderazgo es una responsabilidad compartida, el equipo tiene un propósito específico y bien definido.

- El producto del trabajo es tanto del equipo como del integrante.

- La efectividad se evalúa valorando directamente los productos del equipo. Se hacen evidentes la responsabilidad individual y la del equipo.

- Se reconocen y celebran los esfuerzos individuales que contribuyen al éxito del equipo.

- Se hacen discusiones abiertas para la solución efectiva de problemas.

La interacción grupal ayuda a desarrollar para construir, descubrir, transformar y acrecentar contenidos de naturaleza conceptual, además de socializar en plenitud con las personas que se encuentran en su entorno (Delgado, 2015).

El rol del profesor es de alto desempeño, pues él o ella también conforman horizontalmente el equipo de trabajo colaborativo. Exige tener un buen diseño curricular, didáctica y liderazgo. Todo esto, expone Delgado, basado en el conocimiento adecuado de los contenidos de aprendizaje y del perfil del estudiante. Pues de esta forma guía, atrae y compromete a los estudiantes para asegurar el cumplimiento de las metas propuestas.

\section{BROTES \\ Resultados preliminares}

Algunas ideas empiezan a brotar para el rediseño de la revista Sapientía, como resultantes del análisis de los re- 
ferentes de la categoría y su caracterización, de la encuesta y de insights que surgieron, las cuales ayudaron a tomar decisiones y seguir construyendo la revista. Descubrimos algunas barreras, como resultado del trabajo de campo tomamos algunas decisiones de diseño que compartimos en el siguiente cuadro (Tabla. 2).

\begin{tabular}{|c|c|c|}
\hline ELEMENTOS & BARRERAS & DECISIONES DE DISEÑO \\
\hline $\begin{array}{l}\text { El nombre actual } \\
\text { de la revista } \\
\text { "Sapientía" }\end{array}$ & $\begin{array}{l}\text { - El nombre se usa en categorías } \\
\text { de revistas diversas, muy } \\
\text { diferentes a las científicas } \\
\text { - Percepción del nombre } \\
\text { anticuado, femenino, que } \\
\text { remite al pasado } \\
\text { - Acentuación desconocida "i" } \\
\text { - El nombre no puede cambiarse }\end{array}$ & $\begin{array}{l}\text { - Conservar el nombre, pero } \\
\text { agregarle valor, tornándolo más } \\
\text { genérico, abierto, afirmativo. } \\
\text { - Ubicarlo en el presente, } \\
\text { suprimiendo la tilde }\end{array}$ \\
\hline $\begin{array}{l}\text { El logotipo actual } \\
\text { de la revista } \\
\text { "Sapientía" }\end{array}$ & \begin{tabular}{|l|} 
- Se relaciona su forma \\
(representación gráfica) con \\
otras categorías de productos \\
disímiles a la esperada. \\
- No representa la categoría \\
editorial \\
- No representa al público \\
objetivo al que se busca dirigir
\end{tabular} & $\begin{array}{l}\text { - Rediseño del logotipo o cabezote } \\
\text { con una tipografía, proporción y } \\
\text { estilo más contemporáneos, } \\
\text { funcionales y adaptables. } \\
\text { - Se consideran las características de } \\
\text { las formas que ubiquen a los } \\
\text { lectores en la categoría de revistas y } \\
\text { la representen }\end{array}$ \\
\hline $\begin{array}{l}\text { El enfoque del } \\
\text { público objetivo }\end{array}$ & $\begin{array}{l}\text { - Al inicio de la revista (2015) Se } \\
\text { plantea para un público } \\
\text { objetivo determinado, más } \\
\text { adulto, cerrado o } \\
\text { especializado (profesores } \\
\text { investigadores) y después, al } \\
\text { pasar el tiempo (2017), se } \\
\text { descubren otras necesidades } \\
\text { u oportunidades para dirigirse } \\
\text { a los semilleros (estudiantes } \\
\text { potenciales semilleristas) y } \\
\text { decide enfocarse en este otro } \\
\text { público en forma más abierta } \\
\text { e inclusiva. Todo esto sin } \\
\text { considerar hasta el momento } \\
\text { cambios en el diseño de la } \\
\text { revista }\end{array}$ & $\begin{array}{l}\text { - La nueva personalidad de la marca } \\
\text { debe identificarse con los usuarios } \\
\text { directos, los objetivos y el } \\
\text { contenido } \\
\text { - Se abre el espectro de usuarios } \\
\text { directos e indirectos a comunicar } \\
\text { - Reajuste en la función de } \\
\text { comunicación y misión de la } \\
\text { revista }\end{array}$ \\
\hline El tipo de & $\begin{array}{l}\text { - Es demasiado extenso y } \\
\text { pesado el contenido de cada }\end{array}$ & $\begin{array}{l}\text { - Crear una nueva anatomía } \\
\text { editorial que garantice incluir otro }\end{array}$ \\
\hline contenido & $\begin{array}{l}\text { artículo } \\
\text { - Surgen percepciones } \\
\text { negativas asociadas } \\
\text { - Falta de unidad, calidad y } \\
\text { efectividad de gráficos de los } \\
\text { artículos proporcionados. } \\
\text { - Parece más el contenido de } \\
\text { un cuaderno académico que el } \\
\text { de una revista }\end{array}$ & $\begin{array}{l}\text { tipo de información } \\
\text { - Introducir en el contenido de la } \\
\text { revista temas de interés real para } \\
\text { los usuarios } \\
\text { - Reducir el contenido de los } \\
\text { artículos para hacerlo asimilable e } \\
\text { interesante para los usuarios } \\
\text { - Incluir a los usuarios en la creación } \\
\text { de portada y en la generación de } \\
\text { contenido } \\
\text { - Dar protagonismo a los autores } \\
\text { (rostros) y a sus experiencias como } \\
\text { - semilleristas } \\
\text { - Determinar un concepto creativo } \\
\text { - Agra el nuevo posicionamiento } \\
\text { Agregar color, jerarquía y estilo }\end{array}$ \\
\hline $\begin{array}{l}\text { Desconocimiento } \\
\text { de la publicación }\end{array}$ & $\begin{array}{l}\text { - Difícil acceso a la información } \\
\text { - Percepciones negativas } \\
\text { asociadas } \\
\text { - Poca o nula difusión } \\
\text { - Poco interés de los } \\
\text { estudiantes a este tipo de } \\
\text { publicaciones }\end{array}$ & $\begin{array}{l}\text { - Crear una estrategia de } \\
\text { comunicación de lanzamiento y } \\
\text { difusión de la revista }\end{array}$ \\
\hline
\end{tabular}

Tabla 2. Resultados preliminares Fuente: elaboración propia
Todas estas decisiones de diseño han venido apoyando el desarrollo de nuestros objetivos específicos con acciones que el semillero se plantea cada semestre, y seguirán haciéndolo a medida que se cumplan las etapas del proyecto de investigación en curso, iniciado en el año 2020.

\section{DISCUSIÓN}

Para finalizar y complementar lo compartido en diferentes segmentos de este artículo, queremos compartir algunos temas con potencial y/o que aún están en consideración para su desarrollo, vinculación y análisis. Esta discusión abarca desde las características del producto, hasta la forma de abordar los procesos formativos de investigación y de diseño.

- Se supone una ventaja u oportunidad para el reposicionamiento de la revista Sapientía de su categoría en el mercado. Pues de las pocas revistas indagadas a nivel nacional, observamos que guardan rigurosidad científica y hay muy pocas que estén dirigidas a resultados de semilleros de investigación.

- El tipo de contenido que se propone está mediando contrastes en la comunicación: entre lo científico, el diseño de información y el dinamismo, vitalidad e informalidad, que puede representar el conocimiento para un estudiante universitario. Representa esto una oportunidad para innovar en las publicaciones de esta categoría, sin dejar de cumplir con los requerimientos de publicación.

- Se plantea la necesidad de "Humanizar la revista", dada la naturaleza del contenido y el público objetivo de la publicación. Lo que hace que se traduzcan nuevas formas estilísticas en la visualización de la información.

- El reto presente es traducir en rutas o estrategias transmedia, cada edición de la revista para hacerla interactiva y para que funcione como una revista digital (e-zine o e-journal) efectivamente. 
- El rediseño de la revista Sapientía es una oportunidad para mostrar la variedad de aportes que el Diseño Visual puede dar a los procesos de gestión del conocimiento institucional.

- La falta de entendimiento o enfoque del hallazgo y la comprensión lectora de los temas indagados, en un principio, puede alejar a los estudiantes del gusto por la investigación.

- La integración del Diseño Colaborativo en las dinámicas formativas académicas (planes de estudio) puede incentivar el interés de los estudiantes por la investigación.

\section{REFERENCIAS BIBLIOGRÁFICAS}

Alfonso, F. (2010). Una revisión crítica del proceso de "peer review". Archivos de Cardiología del México, 80(4), 272-282.

Benavides G., E. (2013). Diseño de un prototipo de revista de divulgación académica (Trabajo de grado). Universidad Piloto de Colombia. Bogotá, Colombia.

Brown, T. (2009). Change by design - how design thinking transforms organizations and inspires innovation. Harper Collins.

Buzan, T. (1996). El libro de los mapas mentales. Editorial Urano.

Caldwell C. y Zapaterra, Y. (2014). Diseño editorial: periódicos y revistas / medios impresos y digitales. Editorial Gustavo Gili.

Corte, E. E. (2009). Investigación basada en el diseño: un enfoque prometador para cerrar la brecha entre la teoría y las prácticas educativas. Ponencia magistral para el $\mathrm{X}$ Congreso Mexicano de Investigación Educativa. Veracruz, Septiembre 21-26, 2009.
Delgado, K. (2015). Aprendizaje colaborativo: teoría y práctica. Magisterio Editorial.

Frascara, J. (2005). Diseño gráfico para la gente: comunicaciones de masa y cambio social. Argentina. Ediciones Infinito.

Gibelli, T. (2014). La investigación basada en diseño para el estudio de innovación en educación superior que promueve la autorregulación del aprendizaje utilizando TIC. Congreso Iberoamericano de Ciencias, Tecnología, Innovación y Educación, 2-16.

González, Ma P., Silva, M. y Cornejo, J. M. (1996). Equipos de trabajo efectivos. EUB.

Guillamón, A. (2006). La edición de revistas científicas: directrices, criterios y modelos de evaluación. Fundación Española para la Ciencia y la Tecnología.

Hernández C., M.P. (2010). Guía de diseño editorial para revistas de divulgación académica. Especialización en edición de publicaciones Escuela Interamericana de Bibliotecología Universidad de Antioquia. Medellín, Colombia.

Ruíz Herrera, D., Paz Enrique, L. E., y Hernández Alfonso, E. A. (2018). Diseño de la revista científica electrónica "Investigación Multimedia" . Serie Bibliotecología y Gestión de Información, 105(2018), 1-34.

Kastika, E. (2021). Qué es el pensamiento de diseño. Podcast. Recuperado de: https://open. spotify.com/episode/7J4X78viky0tpjG3 gPMcVW?si=QpG9X5AmTlyljDDiQuc sqA \&nd $=1$

Lesley, J. (2013). The modern magazine: visual journalism in the digital era. Laurence King Publishing.

López V., M. (2009). Guía de estilo editorial para obras académicas. Ediciones del Ermitaño. Centro 
Regional de Investigaciones Universitarias. Universidad Nacional Autónoma de México.

López, M. y Cordero, G. (2005). Un intento por definir las características generales de las revistas académicas electrónicas. Razón y Palabra, 43, pp. 1-31.

Lupton, E. (2017). Intuición, acción, creación. Graphic design thinking. Editorial Gustavo Gili.

Rodgers Paul, M.A. (2012). Métodos de investigación para el diseño de producto. Blume.

Romero-Ariza, M. (2014). Uniendo investigación, política y práctica educativas: dbr, desafíos y oportunidades. Magis, Revista Internacional de Investigación en Educación, 7(14), 159-176.

Sabino, C. (1992). El proceso de investigación. Editorial Papano.

Sanders, E. \& Stappers, P. (2008). Co-creation and the new landscapes of design. CoDesign. 4, 5-18. 10.1080/15710880701875068.

The Design-Based Research Collective (2003). Designbased research: an emerging paradigm for educational inquiry. Educational Researcher, 32(1), 5-8.

Visocky O Grady Jenny \& Visocky O Grady Ken (2018). Manual de investigación para diseñadores Blume.

\section{AUTORES}

Mónica Peláez Montoya: docente del programa Diseño Visual de la Facultad de Ciencias Sociales y Humanas de la UNIAJC. Diseñadora Gráfica, Pedagoga Visual. Especialista en Educación Artística. Maestrante en Educación. Fotógrafa aficionada. Correo: mpelaezm@profesores.uniajc.edu.co
Keyla Tatiana Tejada Pineda: estudiante de Diseño Visual e integrante del semillero Alografía adscrito a la Facultad de Ciencias Sociales y Humanas de la UNIAJC.Correo: kttejada@estudiante.uniajc.edu.co

Angie Melissa Tobar Bolaños: estudiante de Diseño Visual e integrante del semillero Alografía adscrito a la Facultad de Ciencias Sociales y Humanas de la UNIAJC. Correo: amtobar@estudiante.uniajc.edu.co

Lina Marcela Tejada Pineda: estudiante de Diseño Visual e integrante del semillero Alografía adscrito a la Facultad de Ciencias Sociales y Humanas de la UNIAJC. Correo: Imtejada@estudiante.uniajc.edu.co 